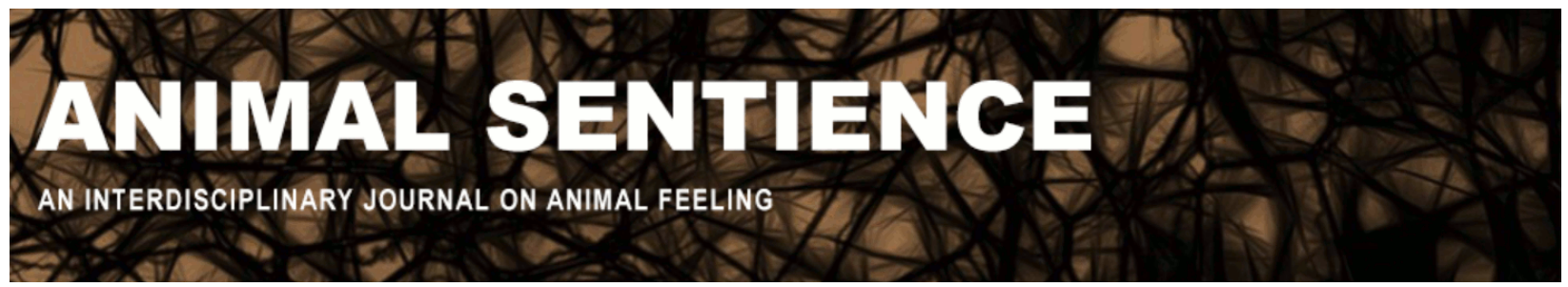

Baker, Liv (2019) Is knowing enough to change human attitudes and actions?. Animal Sentience 25(4)

DOI: $10.51291 / 2377-7478.1439$

Date of submission: 2019-05-10

Date of acceptance: 2019-05-15

(c)

This article has appeared in the journal Animal

Sentience, a peer-reviewed journal on animal

cognition and feeling. It has been made open access,

free for all, by WellBeing International and deposited

in the WBI Studies Repository. For more information,

please contact

wbisr-info@wellbeingintl.org.

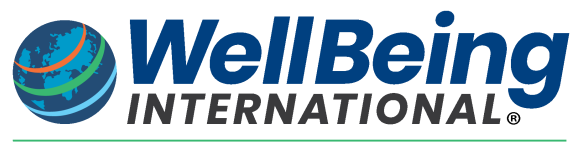

SOLUTIONS FOR PEOPLE, ANIMALS AND ENVIRONMENT 


\title{
Is knowing enough to change human attitudes and actions?
}

Commentary on Marino \& Merskin on Sheep Complexity

\author{
Liv Baker \\ Animal Behavior and Conservation Program, Hunter College, New York, NY, USA
}

\begin{abstract}
Marino \& Merskin present evidence on key aspects of cognition, such as theory of mind, learning, emotional valence, and sociality, to make a convincing argument that sheep are due consideration as individual sentient beings. With this information, what will it take to produce a real, meaningful shift in our attitudes and actions towards other animals, including a species as disadvantaged as sheep? What else do we need to know?
\end{abstract}

Liv Baker is a conservation behaviorist and an expert in wild animal welfare. Liv's work explores similar patterns of well-being seen across the animal kingdom. Her research includes elephants, cetaceans, primates, arachnids, rodents, macropods, and pigs. Liv teaches at Hunter College, CUNY, and is the research director with Mahouts Elephant Foundation, UK. $\underline{\text { Website }}$

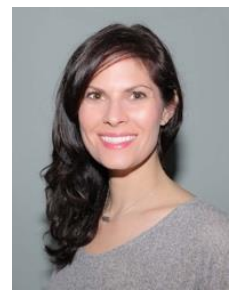

The very moment I began to write this commentary on Marino \& Merskin (2019) (M\&M), I received a news notification that 15 sheep in France have been enrolled as students at a primary school to offset falling numbers in the student population. The sheep were enrolled with birth certificates, and given names, such as Baa-bete, and Saute-Mouton ${ }^{1}$. This occurrence is rife with the contradictions and the disingenuousness with which we treat non-human animals. It exemplifies the stereotypes of sheep that M\&M lay bare in their target article -- docility, passivity, objects of human use; and (ironically in this school context) unintelligence.

Our species has done much to separate itself from other animals. We have long domesticated animal species, artificially selecting traits to serve human values. The degree to which we exploit and harness resources - for food, lodging, technology - has created a parallel version of human life, disparate from all others that inhabit the Earth with us, and in this separation, we have constructed physical and emotional boundaries, discriminating animals as 'other'. We have left other animal species to exist precariously at the whim of human convenience and contradiction, in the shadows of human existence.

M\&M present empirical evidence on key aspects of cognition to make a convincing argument that sheep are due consideration as individual sentient beings. This raises questions, as to why this persuasion is needed, and whether such information can effect real change on behalf of sheep as individuals.

\footnotetext{
${ }^{1}$ https://news.sky.com/story/sheep-registered-as-pupils-in-bid-to-save-classes-at-french-alps-primary-school-
} 11714338 
Let us look briefly at the human relationship with the dog, Canis familiaris, in all its glorious forms (see also Kujala 2017). The domestic dog is reputedly the most privileged nonhuman animal. Americans alone spend close to US\$70 billion on pets each year, averaging about \$1300 for a dog each year. Chinese citizens will spend nearly US\$3 billion on pet-related costs in 2019 (up 50\% from 2016). ${ }^{2}$ As of 2015 , off-leash dog parks have been the fastest growing park type in the US. ${ }^{3}$ There are upwards of 17 research labs dedicated to non-invasive methods of studying dog cognition at major universities worldwide, with at least 12 in the US (e.g. Cook et al., 2019). ${ }^{4}$ Despite the close affection humans have towards dogs, the belief that they have individual personalities and experience emotion, and despite the cognitive evidence to support these sentiments - dogs are still viewed with a utilitarian attitude. About 3.3 million dogs enter the shelter system, with about 700,000 killed each year in the US. Moreover, dogs still remain a 'laboratory animal', commonly used in toxicology tests hin the biomedical field (Hasiwa et al., 2011). In Asia, 30 million dogs a year were slaughtered for consumption as meat in 2016 (Humane Society International, 2016).

The dog evidence shows that even with the species that we individuate almost as much as our own species, we treat other animals as generic commodities. What, then, for the animals we see almost entirely as a mere commodity? The 2016 worldwide estimate for sheep consumption was 550 million (Sanders, 2018). Alongside other food animals, such as pigs, cows, fishes, and chickens, sheep may face the greatest hurdles to achieving ethical considerations. This is in part because of the attributes ascribed to them that have almost entirely effaced their individualities. This is further reflected even in the word 'sheep', whose plural and singular are the same, as for other commodified animals, such as fish, swine, cattle, and poultry. ${ }^{5}$ The fact that a sheep (Dolly) was the first cloned mammal in $1996^{6}$, certainly plays into the overall narrative of sheep as fungible goods. Paradoxically, humans have a tendency to lose sight of animal individuality among highly social, cooperative, and eusocial species (e.g., bighorn sheep, prairie dogs, bees).

How do we individualize sheep in our collective consciousness? Cross-cultural studies show that humans get better at perceiving facially expressed emotions with increased exposure and familiarity (Elfenbein and Ambady, 2003; Reyes et al., 2018.) Moreover, empathy and compassion across races and cultures is higher among more diverse communities (e.g, Sidanius et al., 2008). Thus, the opportunity to see others, and to see them as individuals, is important for developing meaningful and enduring ethical attitudes. Yet with captive Asian elephants, scientific knowledge, education, and even the high valuing of the species has gone only so far in reducing harm. A comprehensive welfare assessment of Asian elephants (in Thailand) suggests that while welfare conditions for tourist elephants have improved, the number of elephants who experience the poorest welfare conditions has increased (Schmidt-Burbach, 2017).

\footnotetext{
${ }^{2}$ http://theconversation.com/americans-spend-70-billion-on-pets-and-that-money-could-do-more-good-102467

${ }^{3}$ https://www.tpl.org/media-room/dog-parks-lead-growth-us-city-parks\#sm.0001q5tkt0m9idzt11v828qfycncg

4 https://dogcog.unl.edu/other-dog-labs

5 https://www.theguardian.com/notesandqueries/query/0,-197456,00.html

${ }^{6}$ https://dolly.roslin.ed.ac.uk/facts/the-life-of-dolly/index.html
} 
To demonstrate the intelligence, complexity, and individuality of sheep, M\&M review the evidence of cognitive capacities such as theory of mind, learning, emotional valence, and sociality. But especially with a species as disadvantaged as sheep, what will it take to induce a real, meaningful shift in our attitudes and actions? What else do we need to know?

\section{References}

Humane Society International (2016) Asia's Dog Meat Trade: FAQs

Cook, Peter; Prichard, Ashley; Spivak, Mark; and Berns, Gregory S. (2018) Jealousy in dogs? Evidence from brain imaging. Animal Sentience 22(1)

Elfenbein, H.A., and Ambady, N. 2003. When familiarity breeds accuracy: cultural exposure and facial emotion recognition. Journal of Personality and Social Psychology, 85(2), 276290.

Hasiwa, N., Baily, J., Clausing, P., Daneshian, M., et al. 2011. Critical evaluation of the use of dogs in biomedical research and testing in Europe. ALTEX. 28(4),326-40.

Kujala, Miiamaaria V. (2017) Canine emotions as seen through human social cognition. Animal Sentience 14(1)

Marino, Lori and Merskin, Debra (2019) Intelligence, complexity, and individuality in sheep. Animal Sentience 25(1)

Reyes, B.N., S.C. Segal, Moulson, M.C. 2018. An investigation of the effect of race-based social categorization on adults' recognition of emotion. PLOS ONE.

Sanders, Bas (2018) Global Sheep Slaughter Statistics and Charts. Faunalytics.

Schmidt-Burbach, J. (2017). Taken for a ride: The conditions for elephants used in tourism in Asia, World Animal Protection (WAP).

Sidanius, J., Levin, S., van Laar, C., and Sears, D.O., 2008. The Diversity Challenge: Social Identity and Intergroup Relations on the College Campus. Russell Sage Foundation. NY, USA. 